\title{
Correction
}

\section{Correction: Saraf-Sinik et al., Motion Makes Sense: An Adaptive Motor-Sensory Strategy Underlies the Perception of Object Location in Rats}

In the article "Motion Makes Sense: An Adaptive Motor-Sensory Strategy Underlies the Perception of Object Location in Rats" by Inbar Saraf-Sinik, Eldad Assa, and Ehud Ahissar, which appeared on pages 8777-8789 of the June 10, 2015 issue, several errors were discovered and are corrected below.

1. In Figure 8, the following corrections were made: $A, p$ values truncated at " $p=1$," as required when applying a Bonferroni correction; $C, H$, and $I$, legends' abbreviations for the word "Arrangement" rewritten in a consistent format; $G$, the label of $x$-axis aligned to the middle; and $I$, the label of $x$-axis changed from " $\dot{\theta}_{W, \max }(\mathrm{deg} / \mathrm{s})$ " to " $\dot{\theta}_{W}(\mathrm{deg} / \mathrm{s}) . "$
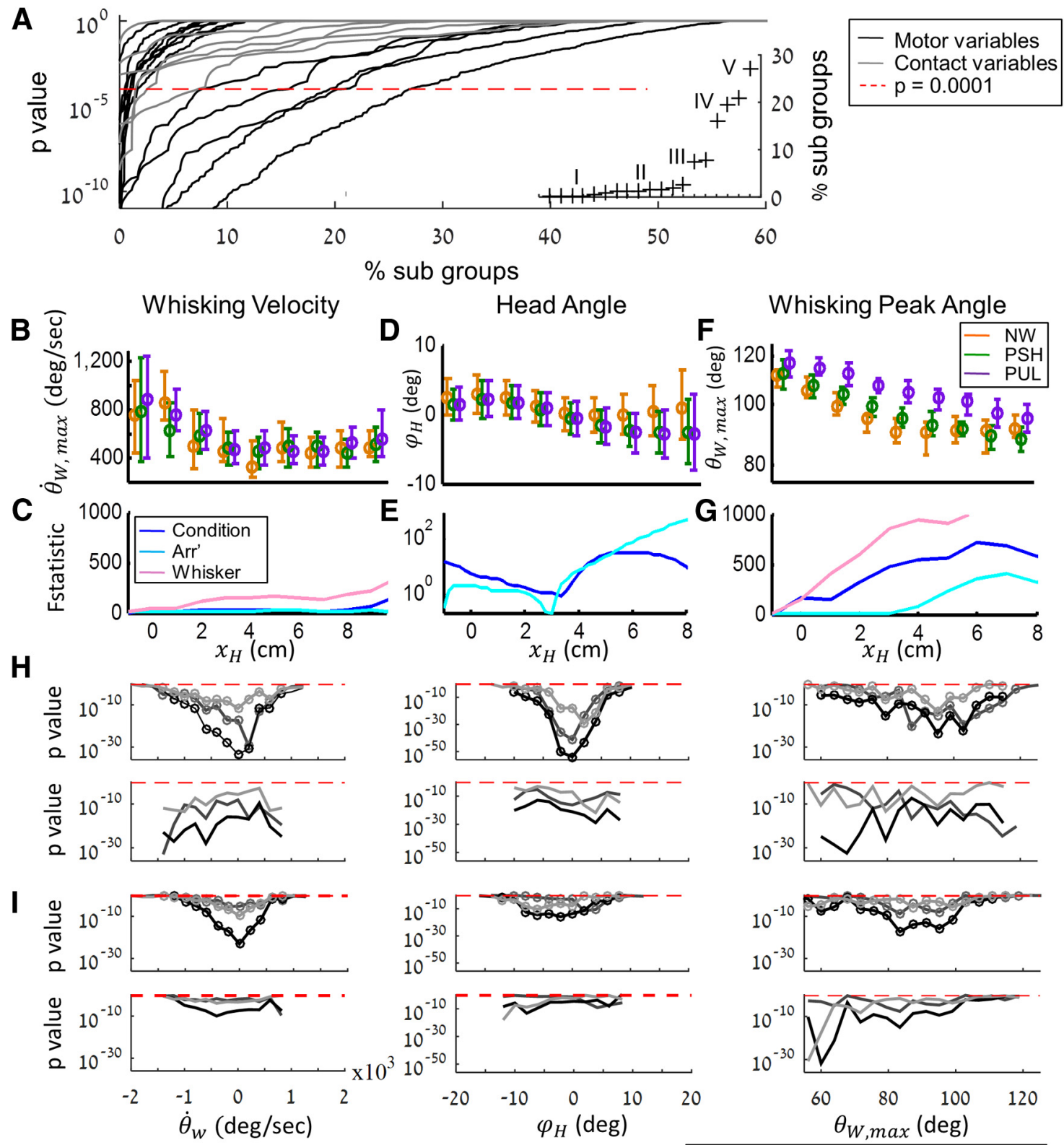

\begin{tabular}{llll}
\hline Arr' 2-Arr' 3 & $-0-$ & Arr' 1 - Arr' 2 & $-0-$ \\
$p=0.05$ & -- & Arr' 1 - Arr' 3 & $-0-$
\end{tabular}

Figure 8. 
Table 3. All analyzed variables sorted by invariance ${ }^{a}$

\begin{tabular}{|c|c|c|c|c|c|}
\hline Group & Variable & $C P$ & No. of sub groups & Mean samples/sub group & Mean samples/group \\
\hline \multirow[t]{4}{*}{ I } & $T_{W, \text { ret }}$ & 0.10 & 371 & 135.94 & 109.36 \\
\hline & $T_{C_{c}}$ & 0.10 & 50 & 36.26 & \\
\hline & $r_{c_{s}}^{c_{s}}$ & 0.10 & 33 & 32.1 & \\
\hline & ${ }^{s} \varphi_{H, C_{S}}$ & 0.10 & 50 & 36.26 & \\
\hline \multirow[t]{9}{*}{ II } & $T_{W, \text { prt }}$ & 0.60 & 376 & 134.51 & 131.47 \\
\hline & $A_{W, \text { ret }}$ & 0.80 & 371 & 135.94 & \\
\hline & $A_{W, \text { prt }}$ & 1.10 & 376 & 134.51 & \\
\hline & $\overline{\dot{\theta}}_{W, \text { ret }}$ & 1.20 & 371 & 135.94 & \\
\hline & $\overline{\dot{\theta}}_{W, p r t}$ & 1.30 & 376 & 134.51 & \\
\hline & $r_{C_{L}}$ & 1.60 & 49 & 39.2 & \\
\hline & $\dot{\theta}_{W, \max }$ & 1.70 & 376 & 134.51 & \\
\hline & $\dot{\theta}_{W, \min }$ & 1.80 & 371 & 135.94 & \\
\hline & $\varphi_{H, C_{1}}$ & 2.70 & 59 & 46.29 & \\
\hline \multirow[t]{2}{*}{ III } & $T_{C_{1}}$ & 7.40 & 59 & 46.29 & 111.45 \\
\hline & $\dot{y}_{H}$ & 7.80 & 96 & 151.49 & \\
\hline \multirow[t]{3}{*}{ IV } & $y_{H}$ & 15.90 & 96 & 151.56 & 141.26 \\
\hline & $\theta_{W, \min }$ & 19.60 & 371 & 135.94 & \\
\hline & $\dot{x}_{H}$ & 20.90 & 96 & 151.49 & \\
\hline V & $\theta_{W, \max }$ & 27.10 & 376 & 134.51 & 134.51 \\
\hline
\end{tabular}

${ }^{a} \mathrm{CP}$, "crossing point" (Fig. 8A): percentage of subgroups below $p=0.0001$.

2. On p. 8786, in Table 3, the second parameter in group IV: changed from " $\dot{\theta}_{W, \text { min }}$ " to " $\theta_{W, \text { min }}$." In the footnote, "Figure $5 A$ " changed to "Figure $8 A$."

3. On page 8786, right column, 11 lines from the bottom, Figure $8 B$ changed to Figure $7 B$. The corrected sentence is: Accordingly, these variables were kept invariant during early localization (Fig. $8 B-E$ ), which indeed maintained the coding power of $\Delta t_{\text {onset }}$ (Fig. $7 B$ ).

These corrections do not affect the main conclusions and interpretations of the paper. The errors have been corrected on the online PDF version.

DOI:10.1523/JNEUROSCI.3147-15.2015 